\title{
The Relationships of Soft Systems Methodology (SSM), Business Process Modeling and e- Government
}

\author{
Dana Indra Sensuse \\ Faculty of Computer Science \\ University of Indonesia \\ Depok, Indonesia
}

\author{
Arief Ramadhan \\ Faculty of Computer Science \\ University of Indonesia \\ Depok, Indonesia
}

\begin{abstract}
Government have emerged in several countries. Because of many aspects that must be considered, and because of there are exist some soft components in e-Government, then the Soft Systems Methodology (SSM) can be considered to use in eGovernment systems development process. On the other hand, business process modeling is essential in many fields nowadays, as well as in e-Government. Some researchers have used SSM in e-Government. Several studies that relate the business processes modeling with e-Government have been conducted. This paper tries to reveal the relationship between SSM and business process modeling. Moreover, this paper also tries to explain how business process modeling is integrated within SSM, and further link that integration to the e-Government.
\end{abstract}

Keywords- Soft Systems Methodology; Business Process Modeling; e-Government.

\section{INTRODUCTION}

e-Government is the use of Information Technology (IT) by public sector organizations [1]. The main orientation of eGovernment is the accessibility of information by the public, rather than financial income, as implied in [1].

Because the target of e-Government is the public sector, then the e-Government systems are generally built based on the web technology. This technology is used because it has ability to reach people quickly and widely. Therefore, the eGovernment can be associated as an attempt to put the public administration online. This means, e-Government is not simply replace all the equipment in the public sector, from a typewriter to a computer. e-Government is more than that. e-Government move the whole system along with existing business processes within the public sector to the online world.

Because of its relation with IT, then most people thought that e-Government is part of computer science. However, eGovernment has become an emergent multidisciplinary field of research [2]. In addition to computer science, there are many other scientific fields in e-Government, for example public administration, management, politics, socio culture etc.

It is revealed in [2], that although theoretical ground is still under construction, e-Government certainly qualifies as a legitimate emerging scientific discipline. It also revealed in [2], that as technological innovations are continuously hitting the market, the frontiers of the e-government discipline are moving and its multidisciplinary nature confirmed [2].

Currently the development of e-Government systems have proliferated in several countries, both in developing countries and developed countries. It is stated in [3], that e-government is a useful tool for modernizing the state given that it enables government to offer higher-quality services to citizens and provide those services in a more efficient, effective, and transparent way.

Heeks in [1] says that e-Government is also an information system, but it is enriched with various aspects, such as the management aspects, political aspects, economical aspects and others. These aspects have to be considered by developers when developing an e-Government system.

Because of many aspects that must be considered, then the e-Government system development process can be very complex. These aspects cannot be observed separately, but should be observed as a whole, where there is interaction in it. Such characteristics can be solved using systems thinking.

e-Government is a socio-technical system that consists of soft components and hard components [1]. It could be argued that the soft component is the people who are involved in eGovernment, whereas the hard component is the Information Technology (IT) that being used. The management approach of the soft component is likely inspired by social sciences, it tends to be subjective, qualitative, and further highlight by the aspects of humanism [1]. The management approach of the hard component is inspired by engineering science, it tends to be objective, quantitative and further highlight by the technical aspects [1].

It is implied in [1], that the most critical factor to determine whether an e-Government system development fail or not is the soft component. Because of the soft component is very dominating and is tend to be subjective, then we see that one type of systems thinking, that is soft systems thinking, can be used in e-Government system development.

Soft systems thinking do not assume that the world is systemic and well-ordered; on the contrary, it assumes social 
reality to be "problematical", characterized by multiple angles of approaches and perspectives [4]. The understanding of reality is dependent upon the observer, his interpretations and what he chooses to focus on [4].

Some of the methodologies that can be used in soft systems thinking is a meta-synthesis approach used in [5], and Soft Systems Methodology (SSM). In this paper, we choose SSM and try to find the relationships between SSM and others.

\section{Soft Systems Methodology}

Soft Systems Methodology (SSM) was proposed in 1981 by Peter Checkland [6]. As the name implies, SSM is based on soft systems thinking. The picture of SSM can be seen in Fig. 1. SSM consists of seven steps, i.e. (extracted from [6]):

1) The identification of a problem situation that demands attention

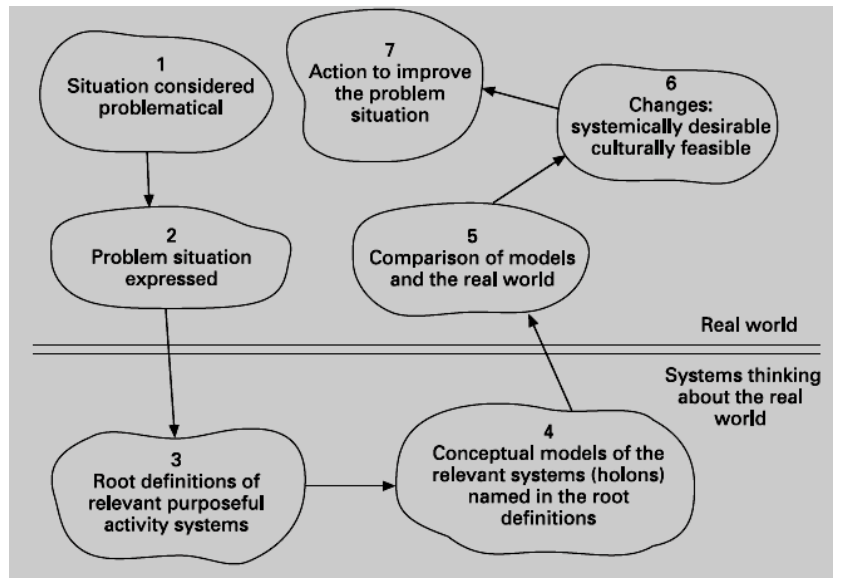

Figure 1. The seven-step of Soft System Methodology (SSM) [6].

2) Problem situation is expressed. The expression can be described using the Rich Picture Diagram. The examples of the Rich Picture Diagram can be seen in Fig. 2.

3) Some relevant human activity systems, potentially offering insight into the problem situation, are selected and from these 'root definitions' are built. In this step, CATWOE analysis is performed. CATWOE stands for Customers, Actors, Transformation process, World view, Owner, and Environmental constraints.

4) Construct conceptual models. This is the most important step in the SSM. Various modes of modeling techniques can be applied at this step.

5) Comparing the conceptual model with the real world. The aim is to provide material for debate about possible change among those interested in the problem situation. This step shows the social processes within the SSM.

6) Making changes to the model by accommodating the interests of several actors involved. Changes should be able to follow the desired model but still possible (feasible) historically, culturally and politically. Changes may include changes in attitudes, structures, or procedures.
7) Perform various activities to implement the model and fix the problem. In this step, the conclusions are drawn and long-term solution is formulated.

SSM has been amended several times. The first change is made in 1990 in the form of "two-strands model" [6]. In this model, were added three types of inquiry, referred to as Analysis 1, 2 and 3 [6]. Analysis 1 considers the intervention itself and the roles of client, problem-solver and problemowners. Analysis 2 is social system analysis. Analysis 3 examines the politics of the problem situation and how power is obtained and used [6].

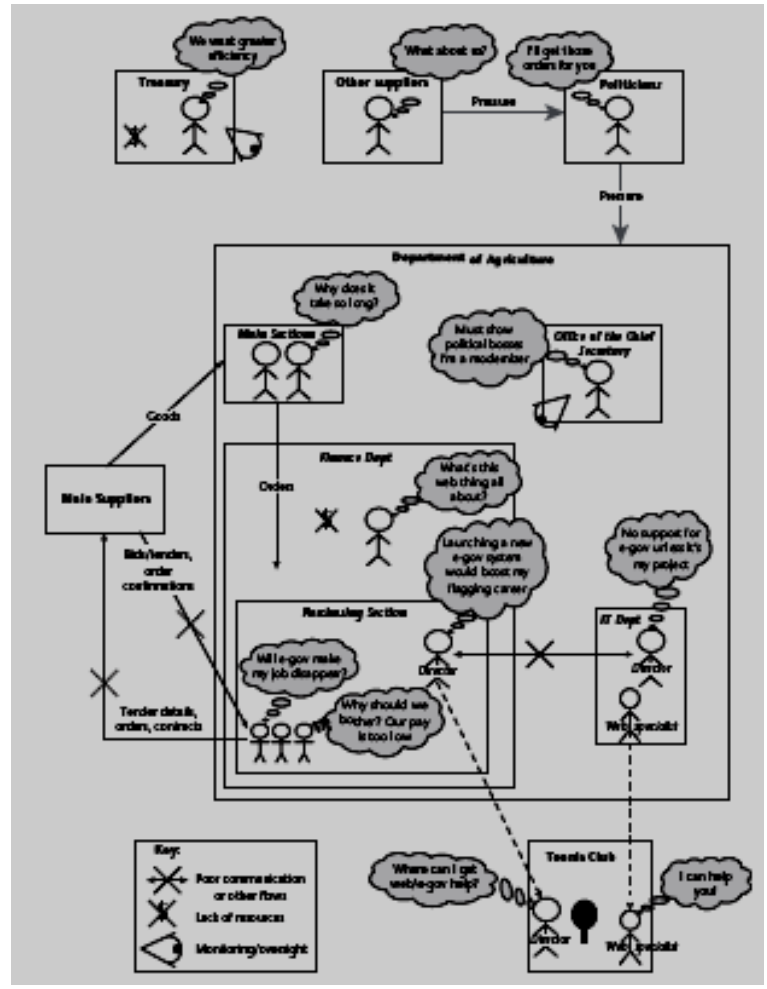

Figure 2. Rich Picture diagram for the system of procurement in the Department of Agriculture [1].

Subsequent change of SSM is made when the original seven-step is merged into just four steps. The new four-step is named as "learning cycle of SSM". Four new steps are [7]:

1) Finding out about a problem situation, including culturally/politically.

2) Formulating some relevant purposeful activity models

3) Debating the situation, using the models, seeking from that debate both :

a) changes which would improve the situation and are regarded as both desirable and (culturally) feasible

b) the accommodations between conflicting interests which will enable action-to-improve to be taken

4) Taking action in the situation to bring about improvement. 
Although the SSM has been amended several times and although Checkland no longer favours it, the representation of SSM as a seven-step, which appeared in 1981, is still frequently used today [6]. Some researchers have used SSM in e-Government, for example can be seen in [24].

\section{Business Process Modeling ANd E-Government}

Business process is characterized by three key words, i.e. activities, linked, and objective. It can be seen from some of the definitions of business process. In [8], it is said that business process is a set of one or more linked procedures or activities which collectively realize a business objective or policy goal, normally within the context of an organizational structure defining functional roles and relationships. Other definition about business process is a set of coordinated tasks and activities, involving both human and system interactions, that will lead to accomplishing a set of specific organizational goals [9]. In [10], it is stated that a business process is a set of related activities or operations which, together, create value and assist organizations to achieve their strategic objectives.

A business process has a clear beginning and end, creating outputs by adding value to inputs [10]. It seems that business process is more likely a function. However, in [11], it was stated that a business process is not the same as a function [11]. It was said in [11], that the people and operations that include in a single business process may come from more than one traditional functional group.

The first stage of the analysis of a business process is concerned with constructing a model of the business process [11]. This "constructing" activity is commonly called business process modeling.

Business process modeling became popular in the context of enterprise reorganization and modernization in the early 1990's [12]. Business process modeling is the visual representation of business processes [13]. Visual representation is usually done in the form of pictures or notations with specific meanings.

Guizzardi et. al. in [14] stated that business process modeling is about the description of sequence of business activities carried out in organizations in order to make them explicit. It is implied in [15], that business process modeling is done for better understanding and analysis about the business process.

It is indicated in [16], that business process modeling can be used to communicate a wide variety of information to a wide variety of audiences. Some audiences who become the main focus are the stakeholders. The graphical nature of business process models can be used as a medium of communication between stakeholders (e.g., executives, developers, and employees) [15]. Business process modeling capabilities as a medium of communication is also revealed in [17].

Currently, there exists some software that can be used to manage the business processes, one of which is SAP [29]. Bider in [30] stated that there are four common views on the process development, i.e.: input/output flow, workflow, agentrelated workflow, and state workflow.
Business process modeling is essential in many fields nowadays, and much research and many initiatives have been proposed in order to facilitate and improve its development [18]. One example of business process modeling activities regarding shipment process of a hardware retailer can be seen in Fig. 3.

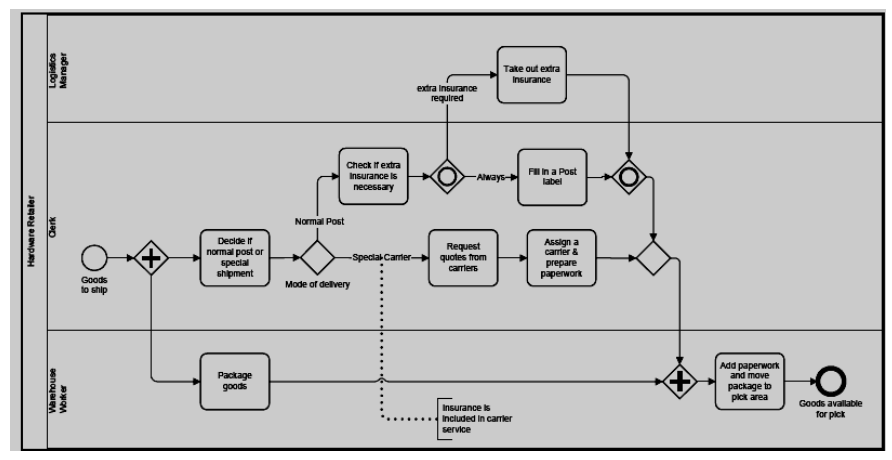

Figure 3. Shipment Process of a hardware retailer [16].

From its name, i.e. "Business Process", and from some of the above explanations, it is impressed that the "business process" is only needed and used within the business sector (private sector). However, there are some studies that link the business process modeling with e-Government (public sector). Several studies that relate the business processes modeling with e-Government can be seen in [25], [26] and [27].

\section{Soft Systems Methodology, Business Process MODELING AND E-GOVERNMENT}

In the section I and II it has been explained that there is a correlation between the SSM with e-Government, that is based on its characteristics, SSM can be used in the development of e-Government systems and that there has been many studies linking SSM with e-Government. Then, in section III it has been revealed that many studies has linked business process modeling with e-Government.

Within this section, we try to describe and propose how SSM can also be associated with business process modeling. In addition, at the same time, we also will reconcile these linkages with e-Government.

In [19], it is shown that the SSM can be associated with business process modeling. In [19], it is implied that the result of business process modeling is depicted using Unified Modeling Language (UML). However, in [19], the process of making business process model with the UML, is depicted as standing outside the SSM. In [19], that being compared with the rich picture diagram is still the conceptual model and not the business process model. It was further shown, that in [19], the process of making business process models with the UML, is conducted separately from the SSM.

In this paper, we propose that the process of making business process model can be fused within SSM. This integration process occurs by placing the process of making business process model into the step 4 of the SSM. So, the conceptual model that originally resulting from that step can be in the form of a business process model, which can be depicted using UML, Business Process Modeling Notation (BPMN) or 
the other. Because of this integration, the result of step 1 (e.g. in the form of surveys results or interviews results), step 2 (e.g. in the form of rich picture diagram) and step 3 (e.g. CATWOE analysis) of the SSM, can be considered by business process modeler to create the business process model in step 4. Henceforth, in step 5, the business process model will be compared with the rich picture diagram of the real-world conditions. It can be seen that in our proposal the making of business process model is integrated within SSM, and not to put it separately. The reason for this integration proposal will be described in the explanation below.

In the conduct of business process modeling there are several obstacles that may be faced. In [28], it was said that defining a business process is a taxing, vexing, and iterative process. Business process modeling is often time-consuming and sometimes involves a certain amount of redundant work because of the similarity between modeling objects [20]. Business process modeling is a complicated process [21].

In addition to these constraints, the business process modeling is also ultimately depends on the modeler who is also a human being. As stated in [22] that the business process model is the result of mapping a business process, and that business process can be either a real-world business process as perceived by a modeler, or a business process conceptualized by a modeler. In this case it appears there are two key words which tend to be subjective and depends on the modeler's perspective, i.e. "to perceive" and "to conceptualize". Because of the subjective tendencies that are "soft" and the fact that business process modeling is very complicated, the SSM is really suitable to this case.

In [23], it is said that building a model of a real business process is a challenging task because:

- Business processes are not always clearly visible as they may go through the whole, often functionally structured organization.

- Written information about business processes is often non-existing or not reliable. The only practical way to obtain reliable information for creating a model of a real business process is by interviewing the people engaged in the process.

From the statement in [23] above, again it is shown that there is a very common thing carried out in SSM, i.e. the interview. In the SSM, the interview, usually carried out in the step 1. This again shows the relationship between SSM with business process modeling.

In addition, business process modeling involves an abstraction from the real-world business process [22]. It is highly compatible with SSM paradigm. Based on Fig. 1, it is shown that the SSM actually also tried to make abstraction of the real-world problems. SSM bring the real-world problems (upper half of the figure) into the conceptual model (bottom half of the figure). The fact that there are similarities between SSM paradigms with business process modeling further strengthen our proposal that the business process modeling can be integrated in the SSM.
Based on the above explanation and based on our explanations in section I, II and III, further, we can see that the SSM, business process modeling and e-Government can be mixed together. In other words, the making of business process model in the development of e-Government system can be done with the SSM.

\section{CONCLUSIONS}

This paper has explained how the business process modeling can be integrated into the SSM. Furthermore, this paper also has described the relationship between the SSM, business process modeling and e-Government. It can be concluded that the SSM can be used in the making of business process models in the development of e-Government system. Some further research can be conducted to enrich this paper, for example the research about how to do the real implementation of the integration of SSM and business process modeling in a country.

\section{REFERENCES}

[1] R. Heeks, Implementing and Managing eGovernment An International Text, London, England : SAGE Publications, 2006.

[2] S. Assar, I. Boughzala, and I. Boydens, "Back to Practice, a Decade of Research in E-Government", in Practical Studies in E-Government : Best Practices from Around the World, S. Assar, I. Boughzala, and I. Boydens, Eds. New York, USA: Springer, 2011.

[3] L. Herrera and J. R. Gil-Garcia, "Implementation of E-Government in Mexico: The Case of Infonavit", in Practical Studies in E-Government : Best Practices from Around the World, S. Assar, I. Boughzala, and I. Boydens, Eds. New York, USA: Springer, 2011.

[4] A. Mirijamdotter, "A Multi-Modal Systems Extension to Soft System Methodology," Ph. D. dissertation, Lulea Tekniska Universitet, Sweden, 1998.

[5] J. Gu and X. Tang, "Meta-Synthesis System Approach To Knowledge Science," International Journal of Information Technology \& Decision Making, vol. 6, no. 3, pp. 559-572, 2007.

[6] M. C. Jackson, System Thinking Creative Holism for Managers, John Wiley \& Sons Ltd, England, 2003.

[7] P. Checkland, "Soft Systems Methodology: A Thirty Year Retrospective," Systems Research and Behavioral Science, vol. 17, pp. S11-S58, 2000.

[8] [Workflow Management Coalition], "The Workflow Management Coalition Specification : Terminology \& Glossary", Document Number WFMC-TC-1011, Document Status - Issue 3.0, 1999.

[9] [Oracle], "Oracle Application Integration Architecture: Business Process Modeling and Analysis", Oracle White Paper, April 2009.

[10] [Australian Government Information Management Office], "The Australian Government Business Process Interoperability Framework", July 2007.

[11] S. J. Childe, P. A. Smart, and A. M. Weaver, "The use of generic process models for process transformation," in Proceedings of the IFIP TC5 WG5.7 international workshop on Modelling techniques for business process re-engineering and benchmarking, pp. 51-60, 1997.

[12] R. von Ammon, C. Emmersberger, F. Springer, and C. Wolff, "EventDriven Business Process Management and its Practical Application Taking the Example of DHL," in Proceedings of the 1st iCEP08 Workshop on Complex Event Processing for the Future Internet, 2008.

[13] M. Ramadan, H. G. Elmongui, and R. Hassan, "BPMN Formalisation using Coloured Petri Nets," in Proceedings of the 2nd GSTF Annual International Conference on Software Engineering \& Applications (SEA 2011), 1997.

[14] R. S. S. Guizzardi, G. Guizzardi, J. P. A. Almeida, and E. Cardoso, "Ontological Foundations for Agent-Oriented Organizational Modeling," in Proceedings of the 3rd International i* Workshop istar08, pp. $37-41,2008$. 
[15] A. Lodhi, V. K"oppen, and G. Saake, "Business Process Modeling: Active Research Areas and Challenges", Department of Technical and Business Information Systems, Faculty of Computer Science, Otto-vonGuericke University, Tech. Rep., Nr : FIN-001-2011, 2001.

[16] [Object Management Group], "Business Process Model and Notation (BPMN) Version 2.0", OMG Document Number : formal/2011-01-03, 2011.

[17] A. Popovic, M. I. Stemberger, and J. Jaklic, "Applicability of Process Maps for Simulation Modeling in Business Process Change Projects," Interdisciplinary Journal of Information, Knowledge, and Management, vol. 1, pp. 109-123, 2006.

[18] A. Koschmider, J. L. de la Vara, and J. Sanchez, "Measuring the Progress of Reference Model-Based Business Process Modeling," in Proceedings of 3rd International Conference on Business Process and Services Computing (BPSC 2010), pp. 218 - 229, 2010.

[19] M. Salahat and S. Wade, "Measuring the Progress of Reference ModelBased Business Process Modeling," in Proceedings of The 5th International Conference on Innovations in Information Technology, 2008.

[20] C. Ren, W. Wang, J. Dong, H. Ding, B. Shao, and Q. Wang, "Towards A Flexible Business Process Modeling And Simulation Environment," in Proceedings of the 2008 Winter Simulation Conference, IEEE Xplorer, pp. 1694-1701, 2008.

[21] R. Lu and S. Sadiq, "A Survey of Comparative Business Process Modeling Approaches," in Lecture Notes in Computer Science, Springer, Vol. 4439/2007, 82-94, 2007.

[22] J. Mendling, "Foundations of Business Process Modeling", in Handbook of Research on Modern Systems Analysis and Design Technologies and Applications, M. R. Syed and S. N. Syed, Eds. Hershey, USA: IGI Global, 2009.

[23] I. Bider, "State-Oriented Business Process Modeling: Principles, Theory and Practice," Ph. D. dissertation, Royal Institute of Technology and Stockholm University, Sweden, 2002.

[24] M. Alrazooqi and R. De Silva, "Mobile and Wireless Services and
Technologies for M-Government Solution Proposal for Dubai Government," WSEAS Transactions on Information Science And Applications, Issue 8, Vol. 7, pp. 1037-1047, 2010.

[25] J. Becker, D. Pfeiffer, M. Rackers, and P. Fuchs, "Business Process Management in Public Administrations - The PICTRUE Approach," in Proceedings of 11th Pacific-Asia Conference on Information Systems, 2007.

[26] O. F. Aydınl, S. Brinkkemper, and P. Ravesteyn, "Business Process Improvement in Organizational Design of E-Government Services", Department of Information and Computing Sciences Utrecht University, Tech. Rep. UU-CS-2007-041, 2007.

[27] P. Liegl et. al., "Modeling eGovernment processes with UMM," Informatica, Vol. 31, pp. 407-417, 2007.

[28] F. Nickols, "The Difficult Process of Identifying Processes : Why it isn't as easy as it sounds," Knowledge and Process Management, Vol. 5, No. $1,1998$.

[29] [www.winshuttle.com]. 2011. "How SAP Users hold the key to Business Process Improvement". http://www.winshuttle.com/WhitePapers/Winshuttle-HowSAPUsersHoldtheKeytoBPI-whitepaper-EN.pdf

[30] I. Bider 2002. "Tutorial on: Business Process Modeling as a Method of Requirements Engineering”. http://www.iceis.org/iceis2007/ Hall_Of_Fame/ ibider/ibider_2002.pdf

\section{AUTHORS PROFILE}

Dana Indra Sensuse. B.Sc in Soil Science (Bogor Agricultural University, Indonesia, 1985), M.Sc in Library and Information Studies (Dalhousie University, Halifax, Canada, 1994), Ph.D in Information Studies (Toronto University, Canada, 2004), Lecturer at University of Indonesia, Head of eGovernment Lab at University of Indonesia.

Arief Ramadhan. B.Sc in Computer Science (Bogor Agricultural University, Indonesia, 2005), M.Sc in Computer Science (Bogor Agricultural University, Indonesia, 2010), Ph.D Student in Computer Science (University of Indonesia), Research Assistant at University of Indonesia. Member of eGovernment Lab at University of Indonesia. 